\title{
OPEN The influence of PI3K inhibition on the radiotherapy response of head and neck cancer cells
}

\author{
Mary Glorieux ${ }^{1}$, Rüveyda Dok $^{1} \&$ Sandra Nuyts ${ }^{1,2 \bowtie}$ \\ Radiotherapy has a central role in the treatment of head and neck squamous cell carcinoma \\ (HNSCC). Activation of the PI3K/AKT/mTOR pathway can decrease the efficiency of radiotherapy \\ via the promotion of cell survival and DNA repair. Here, the influence of PI3K pathway inhibition on \\ radiotherapy response was investigated. Two PI3K inhibitors were investigated and both BKM120 and \\ GDC0980 effectively inhibited cellular and clonogenic growth in $6 \mathrm{HNSCC}$ cells, both HPV-positive as \\ well as HPV-negative. Despite targeted inhibition of the pathway and slight increase in DNA damage, \\ PI3K inhibition did not show significant radiosensitization. Currently only one clinical trial is assessing \\ the effectiveness of combining BKM120 with RT in HNSCC (NCT02113878) of which the results are \\ eagerly awaited.
}

With almost 650,000 diagnoses and 330,000 deaths every year, head and neck squamous cell carcinoma (HNSCC) remains the sixth most prevalent cancer worldwide ${ }^{1}$. Tobacco and alcohol use are known risk factors for HNSCC as well as infection with human papillomavirus (HPV) $)^{2,3}$. Radiotherapy (RT) plays a prominent role in HNSCC treatment next to chemotherapy and surgery ${ }^{4}$. The 5 -year survival proportion for locally advanced HNSCC is only $40-50 \%$ with locoregional recurrence remaining the predominant form of treatment failure, often linked to radioresistance of the tumour ${ }^{5,6}$. It is documented that activation of signal transduction pathways can lead to enhanced DNA repair in tumour cells resulting in a decreased efficiency of $\mathrm{RT}^{7}$.

One of the key pathways involved in resistance to therapies, including resistance to RT, is the phosphatidylinositol 3 kinase (PI3K) - protein kinase B (AKT) - mammalian target of rapamycin (mTOR) pathway ${ }^{8}$. This pathway is involved in three main mechanism of radioresistance namely tumour cell proliferation, hypoxia and intrinsic radioresistance ${ }^{9}$. Furthermore, it is known that this pathway has implications in classic tumorigenic processes such as apoptotic resistance, angiogenesis, invasion and metastases ${ }^{10,11}$. According to The Cancer Genome Atlas (TCGA), activating mutations in the PIK3CA gene are seen in 56\% of HPV-positive and $34 \%$ of HPV-negative HNSCC $^{12,13}$. Mutations in PTEN, AKT or the mTOR complex may lead to constitutive activation of the PI3K/ $\mathrm{AKT} / \mathrm{mTOR}$ pathway, resulting in cell growth and proliferation in the absence of nutrients ${ }^{14,15}$. Therefore, there is intense interest in molecular therapies targeting the $\mathrm{PI} 3 \mathrm{~K} / \mathrm{AKT} / \mathrm{mTOR}$ pathway ${ }^{8}$.

Multiple inhibitors of the PI3K/AKT/mTOR pathway have been developed, of which we selected two to study the efficacy in combination with RT in HNSCC cells ${ }^{13,16}$. Buparlisib, NVP-BKM120 (BKM120), is a novel, selective pan-class $1 \mathrm{PI} 3 \mathrm{~K}$ inhibitor that works via reversible competition to $\mathrm{ATP}^{17-19}$. The anti-proliferative, proapoptotic and anti-angiogenic effects of BKM120 have been proven in a variety of tumour types, irrespective the PIK3CA status ${ }^{20-22}$. Currently six clinical trials are ongoing with BKM120 in HNSCC, of which only 1 involves combination with RT (NCT02113878). Next, the dual PI3K-mTOR inhibitor Apitolisib, GDC0980, is a small molecule inhibitor of class 1 PI3K and mTOR (mTORc1 and mTORc2) synthesized by substituting the indazole in GDC-0941 for a 2-aminopyrimidine ${ }^{16,23}$. Preclinical testing of GDC0980 showed attainable inhibition of the pathway, great potency against a wide variety of cell lines and inhibited tumour growth in vivo ${ }^{24,25}$.

In this study we investigated the response to a pan PI3K inhibitor, BKM120, and a dual PI3K/mTOR inhibitor, GDC0980, in three HPV-positive and three HPV-negative HNSCC cell lines alone and in combination with RT.

\footnotetext{
${ }^{1}$ Laboratory of Experimental Radiotherapy, Department of Oncology, KU Leuven, University of Leuven, 3000 Leuven, Belgium. 2Department of Radiation Oncology, Leuven Cancer Institute, UZ Leuven, 3000 Leuven, Belgium. ${ }^{\boxplus}$ email: sandra.nuyts@uzleuven.be
} 

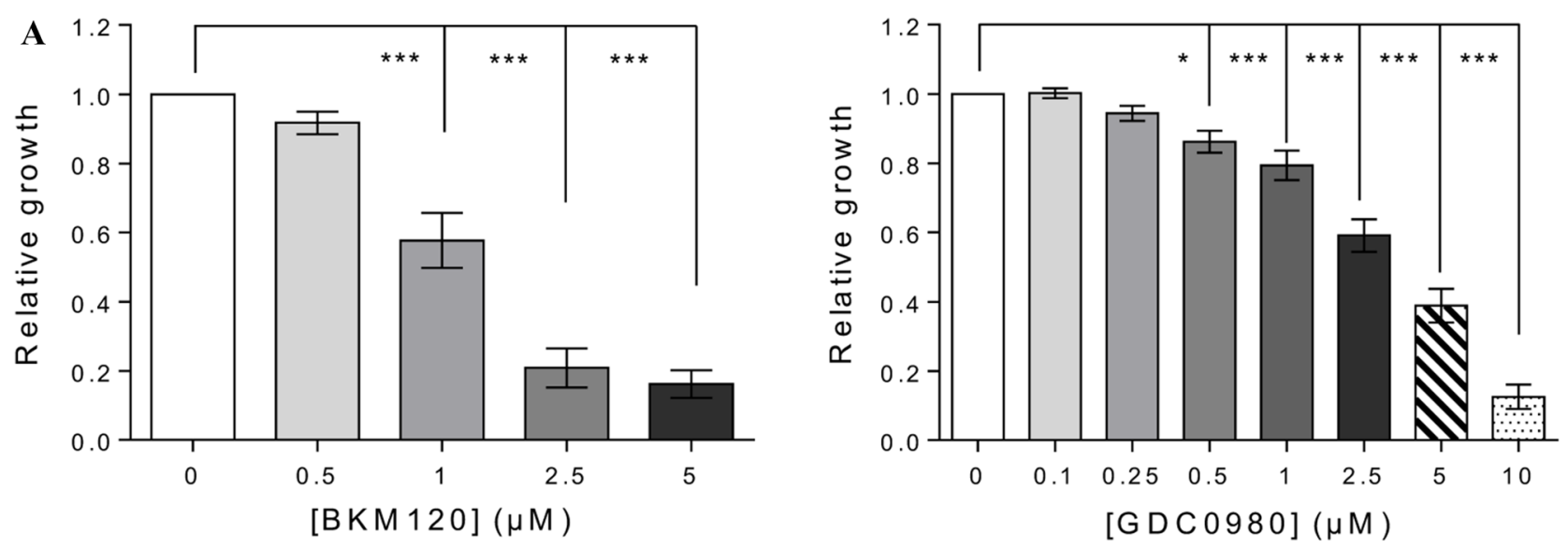

B
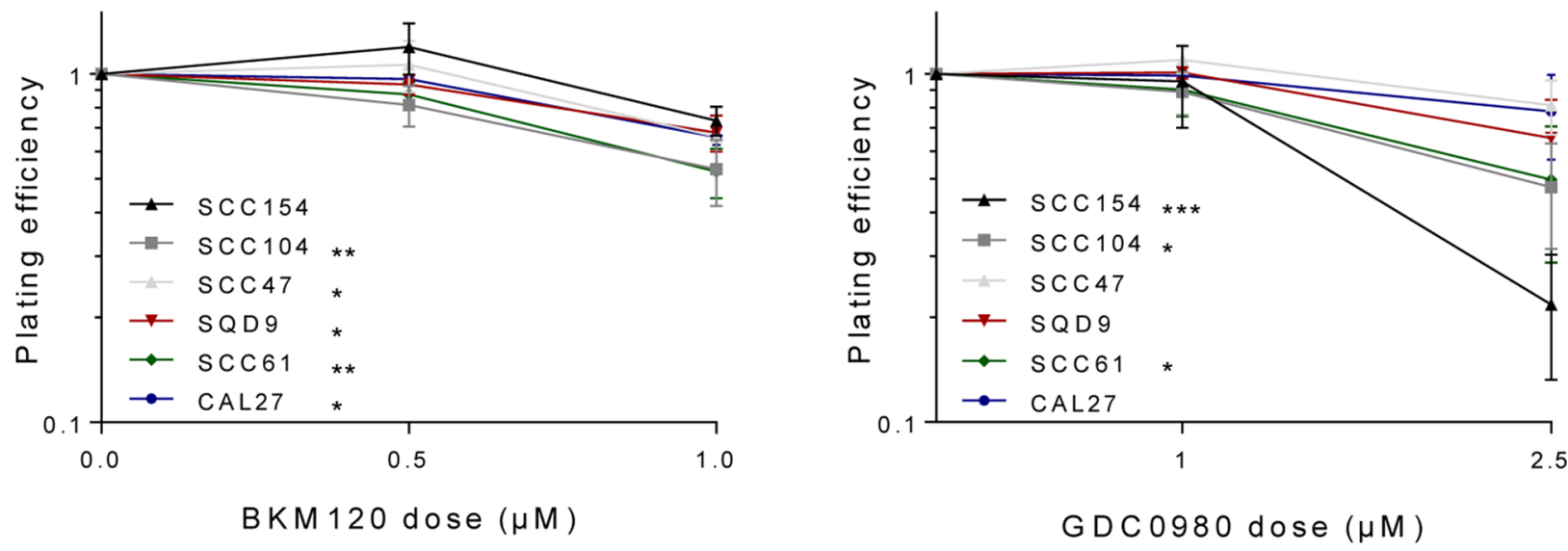

Figure 1. (A) Dose response curves of BKM120 (left) and GDC0980 (right). Cells were treated for $72 \mathrm{~h}$ with the indicated concentrations of BKM120 (left) and for $24 \mathrm{~h}$ with GDC0980 (right). Data is presented as the mean \pm SEM of three HPV-positive cell lines (SCC154, SCC104 and SCC47) and three HPV-negative cell lines (SQD9, SCC61, CAL27) HNSCC cell lines of which 3 independent experiments were performed per cell line. One-way Anova analysis plus posthoc t-testing with Bonferroni correction showed the statistical significant differences. (B) The effect of PI3K inhibition monotherapy on plating efficiency of HNSCC cell lines. Colony assay of HNSCC cells treated with BKM120 for $72 \mathrm{~h}$ (left) or with GC0980 for $24 \mathrm{~h}$ (right). Colony formation was tested in three HPV-positive cell lines (SCC154, SCC104 and SCC47) and three HPV-negative cell lines (SQD9, SCC61, CAL27). Data are represented as the mean plating efficiency \pm SEM for three independent performed experiments. One-way Anova analysis plus posthoc t-testing with Bonferroni correction showed the statistical significant differences.

\section{Results}

The effect of PI3K inhibition alone or in combination with RT on cellular growth and survival. The effect of PI3K inhibition on the growth and survival of HNSCC cells was investigated with the panPI3K inhibitor BKM120 and the dual PI3K and mTOR inhibitor GDC0980 by a short-term survival assay and colony assay (Fig. 1A,B). Both PI3K inhibitors BKM120 $(0-5 \mu \mathrm{M})$ and GDC0980 (0-10 $\mu \mathrm{M})$ effectively inhibited cellular growth dose-dependently in all HNSCC cells (Fig. 1A, Supplementary Fig. 1).

Based on the dose response curves, two concentrations for each of the inhibitors were selected for colony assay. BKM120 (0.5 and $1 \mu \mathrm{M}$ incubated for $72 \mathrm{~h}$ ) and GDC0980 ( 1 and $2.5 \mu \mathrm{M}$ incubated for $24 \mathrm{~h}$ ) monotherapy resulted in decreased colony formation at the highest treated concentrations. In addition, different cell lines showed differences in the sensitivity to the drug (Fig. 1B, Supplementary Fig. 2).

BKM120 $(0.5$ and $1 \mu \mathrm{M}$ ) in combination with RT (given $2 \mathrm{~h}$ before RT for $72 \mathrm{~h}$ ) did not enhance the RT response of the HPV-positive and HPV-negative HNSCC cells (Fig. 2A). Only in the HPV-positive cell line SCC154 a small radiosensitizing effect was seen at $0.5 \mu \mathrm{M}(\mathrm{p}=0.02146)$. Also, GDC0980 $(1$ and $2.5 \mu \mathrm{M})$ in combination with RT (given $2 \mathrm{~h}$ before RT for $24 \mathrm{~h}$ ) did not result in decreased survival of RT treated HNSCC cells (Fig. 2B). In some cell lines, SCC47 and SCC61, even an increase in survival was observed ( $p=0.01989$ for SCC47, $\mathrm{p}=0.006706$ and $\mathrm{p}=3.692^{\star} 10^{-5}$ for SCC61). To assess whether continuous PI3K inhibition is needed for radiosensitization, HNSCC cells were exposed to BKM120 or GDC0980 $(0.1,0.25$ and $0.5 \mu \mathrm{M})$ in combination with RT ( $2 \mathrm{~h}$ before RT) for 2-3 weeks long. However, no radiosensitizing effect was observed (Supplementary Fig. 3). Here again, for some cell lines, there was a statistically significant increase in survival $(\mathrm{p}=0.03756$ and $\mathrm{p}=0.04984$ for CAL27, and $\mathrm{p}=0.006371$ for SCC61). 

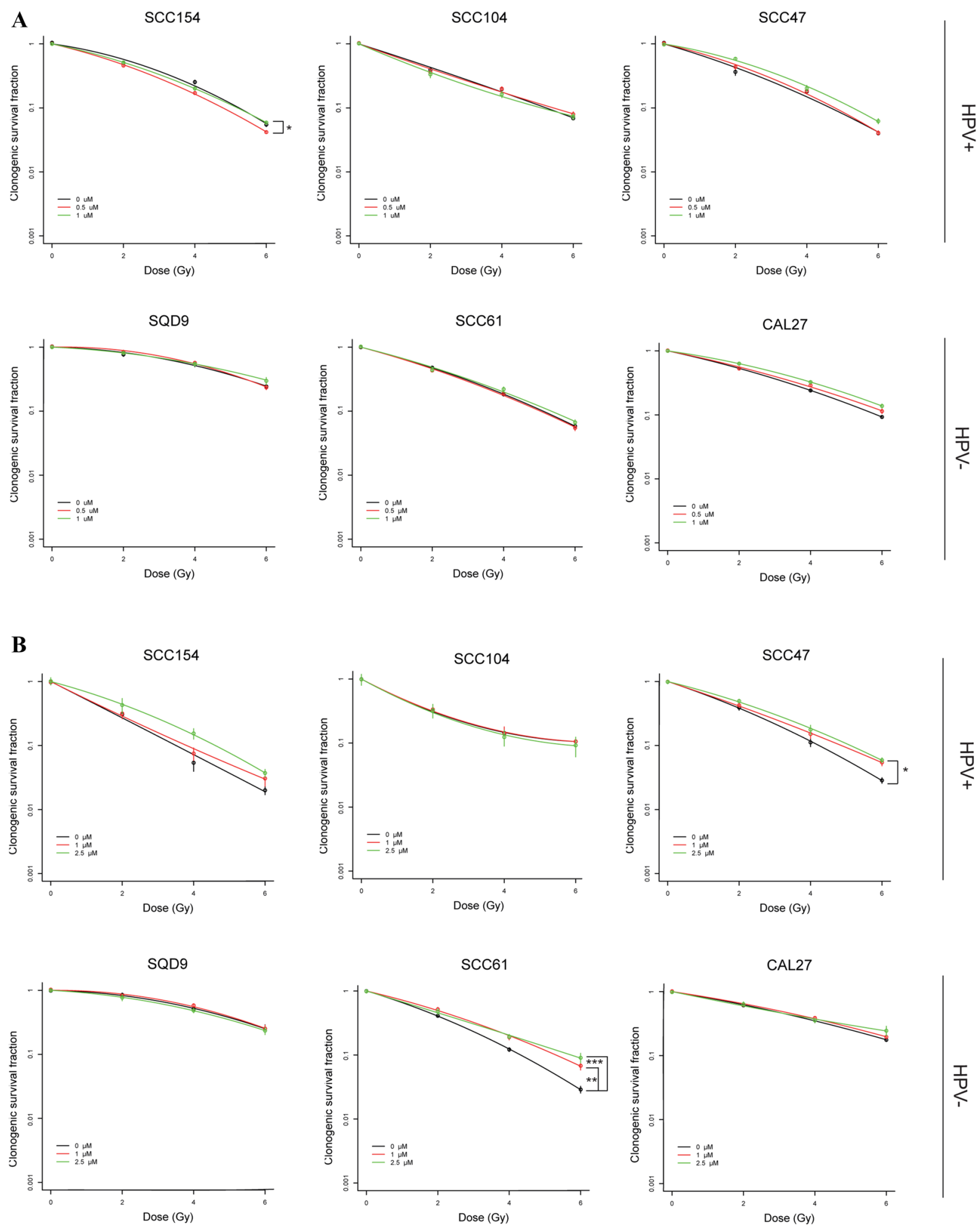

Figure 2. The effect of PI3K inhibition on the clonogenic survival of HNSCC cell lines. (A) Colony assay of HNSCC cells treated with BKM120. Cells were irradiated $2 \mathrm{~h}$ after drug exposure and the drug was removed $70 \mathrm{~h}$ after radiotherapy. (B) Colony assay of HNSCC cells treated with GDC0980. Cells were irradiated $2 \mathrm{~h}$ after drug exposure and the drug was removed $22 \mathrm{~h}$ after radiotherapy. Data are represented as the mean \pm SEM for three independent performed experiments. The fitted curves were compared with Anova analysis to show the statistical significant differences. 
The effect of PI3K inhibition alone or in combination with RT on PI3K/AKT/mTOR pathway. The inhibitory effect of both inhibitors on the PI3K pathway was investigated by immunoblotting key proteins of the pathway in two HPV-positive (SCC154, SCC104) and two HPV-negative (SQD9, SCC61) HNSCC cell lines. Both BKM120 and GDC0980 treatment resulted in inhibition of the PI3K/AKT/mTOR pathway as can be seen by a reduction in phosphorylation of AKT, S6 and 4EBP1 compared to the untreated controls (Fig. 3). Inhibition of PI3K pathway by the PI3K inhibitors was seen in the monotherapy conditions as well as in the combination treatment condition (Fig. 3). A more effective inhibition of pAKT and p4EBP1 could be observed with the dual inhibitor.

The effect of PI3K inhibition alone or in combination with RT on DNA damage response. We assessed whether PI3K inhibition ( $1 \mu \mathrm{M}$ BKM120) alone or in combination with RT ( $2 \mathrm{~h}$ after drug exposure) can induce alterations in cell cycle and gH2AX foci kinetics in HPV-positive SCC104 and HPV-negative SQD9 cells (Fig. 4A, B).

BKM120 monotherapy induced a significant G2/M arrest at $24 \mathrm{~h}$ compared to the baseline condition in the HPV-positive cells $(\mathrm{p}=0.0058)$. When BKM120 was added to RT treatment, this resulted in a significant G2/M arrest after $24 \mathrm{~h}$ in both the HPV-positive (SCC104) and HPV-negative (SQD9) cells ( $\mathrm{p}=0.0021$ and $\mathrm{p}=0.0093$ respectively) compared to the RT conditions. After $72 \mathrm{~h}$ the cells rendered back to the baseline condition (Fig. 4A).

As expected, a significant increase (twofold) in gH2AX foci was seen $2 \mathrm{~h}$ after RT alone in both cell lines $(\mathrm{p}<0.0001)$ (Fig. 4B). The combination of BKM120 and RT did show residual gH2AX foci in the HPV-positive cell line at $24 \mathrm{~h}(\mathrm{p}=0.0454)$. Whereas in the HPV-negative cells no difference in $\mathrm{gH} 2 \mathrm{AX}$ was detected between the RT treatment alone or in combination with BKM120 (Fig. 4B).

\section{Discussion}

Since the relapse rate of locally advanced HNSCC remains very high despite progress made in surgical and radiotherapeutic techniques, molecular targeted drugs have become an appealing strategy to improve treatment response. Unfortunately very few targeted agents advanced to phase 3 clinical trials in combination with $\mathrm{RT}^{26}$. In this paper, we investigated the radiosensitizing potential of $\mathrm{PI} 3 \mathrm{~K} / \mathrm{AKT} / \mathrm{mTOR}$ pathway inhibitors in a panel of six HNSCC cell lines.

Although the PI3K pathway is the most frequently deregulated pathway in HNSCC, scientific literature is replete of contradictory data around the predictive value of PIK3CA status. Several studies showed that mutations in PIK3CA, which lead to PI3K/AKT activation, correlate with response to PI3K inhibition ${ }^{11}$. This in contrast to amplification of PI3K/AKT that has been associated with radioresistance ${ }^{27}$. Alpelisib, the first PI3K inhibitor approved by the FDA, is approved to treat breast cancer patients with PIK3CA-mutation ${ }^{28}$. The selectivity of Alpelisib for PIK3CA-mutated cells has also been seen in HNSCC cells by Keam et al. ${ }^{29}$. However, when we look at BKM120, the study of Kong et al. investigated the effect of BKM120 in PIK3CA hotspot mutated and wild type cell lines and it was concluded that BKM120 exhibit potent activity in both ${ }^{30}$. Since the value of PIK3CA status as predictive biomarker is still unknown according to Anizussaman et al. and we had limited availability of HNSCC cell lines with a PIK3CA mutation, we did not incorporate a PIK3CA-mutated cell line in our experimental set-up ${ }^{15,31-35}$.

Preclinical models showed that BKM120 has a strong anti-proliferative activity in more than 400 cancer cell lines $^{36}$. This is in line with our study in which we see that both PI3K inhibitors showed significant inhibitory effects on proliferation and growth of all the tested cell lines, which were wildtype for PIK3CA. Although, it should be mentioned that the antiproliferative effects of the inhibitors were cell line dependent.

Next, we evaluated whether PI3K inhibition could increase the RT response in HNSCC cells. Although we could demonstrate effective inhibition of the $\mathrm{PI} 3 \mathrm{~K} / \mathrm{AKT} / \mathrm{mTOR}$ pathway, no radiosensitizing effect was observed despite testing different time schedules. This is in concordance with the study of Blas et al. in which different time schedules were tested and no significant difference in HNSCC was observed ${ }^{37}$. In addition, combining RT with BKM120 did not modify the radiation response and even reduced the effectiveness of BKM120 alone in inhibiting the PI3K/AKT/mTOR pathway ${ }^{37}$. In literature, other dual PI3K/mTOR inhibitors, namely PF-05212384, PF-04691502 and the $\beta$-sparing PI3K inhibitor GDC-0032, showed significant radiosensitizing effects both in vitro and in vivo in HNSCC ${ }^{38-40}$. Moreover, BKM120 demonstrated radiosensitizing effects in other cancer types $^{41-43}$. However, the radiosensitizing effect of BKM120 in HNSCC has only been described mainly in vivo in two studies ${ }^{44,45}$. In these studies the tumour microenvironment seems to play an important role since the PI3K/ $\mathrm{AKT} / \mathrm{mTOR}$ pathway plays a role in $\mathrm{O} 2$-consumption thereby contributing to tumour hypoxia ${ }^{46}$. The importance of oxygenation has also been seen in the study of Burrows et al. where PI3K inhibition with GDC0941 in combination with RT reduced clonogenic survival only under anoxia conditions ${ }^{47}$. It has also been shown that BKM120 remodeled tumour vasculature and decreased hypoxia which could explain the radiosensitizing effects seen in vivo but not in vitro as in the study of Blas et al. ${ }^{18,48,49}$. Moreover, recent results published about the phase 1 clinical trial investigating the effect of BKM120 on palliative RT showed that BKM120 indeed reduced tumour hypoxia in NSCLC patients ${ }^{50}$. Studies using GDC0980 are limited to breast cancer and mesothelioma of which none combined GDC0980 with $\mathrm{RT}^{51,52}$.

The influence of the PI3K pathway on DSB repair and DNA damage response is documented in several studies and is one of the major factors for the hypothesis of combining PI3K inhibitors with $\mathrm{RT}^{7,53-55}$. In our study, the combination of BKM120 with RT resulted in a significant but limited presence of residual DNA damage in the HPV-positive cell line. The HPV-positive SCC104 cells treated with RT demonstrated less gH2AX foci than the HPV-negative HNSCC cells which can be ascribed to impairment in DSB repair in HPV-positive HNSCC cells due to decreased activity of both NHEJ and HRR and is in concordance with the knowledge that HPV-positive cells show increased sensitivity to $\mathrm{RT}^{56-59}$. 
A
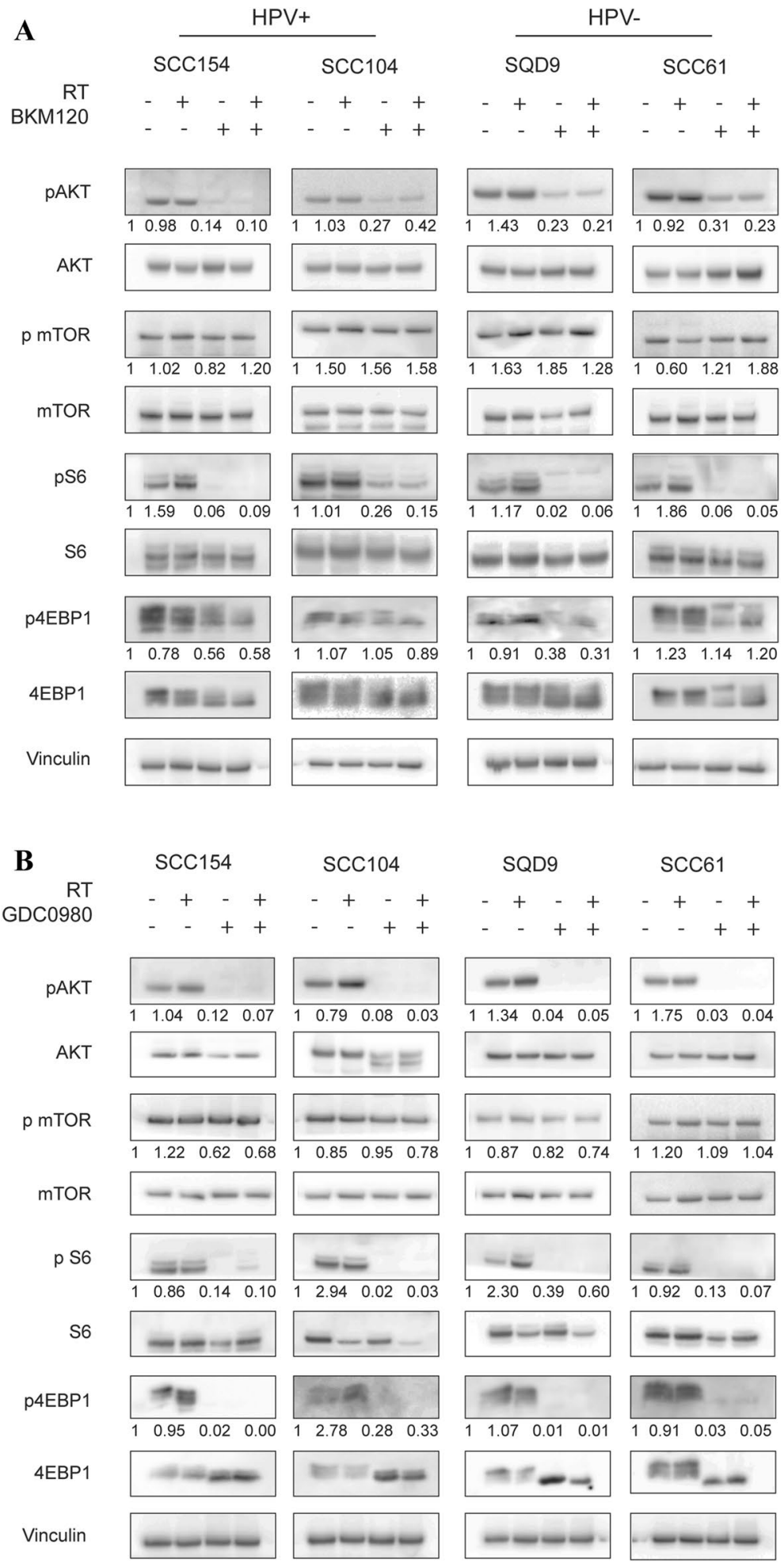

Figure 3. The effect of BKM120 and GDC0980 on the PI3K pathway. (A) Cells were treated with BKM120 $(1 \mu \mathrm{M}) 2 \mathrm{~h}$ before radiotherapy (6 Gy). Control condition cells are treated with equal concentrations of DMSO. After $24 \mathrm{~h}$, total cell lysates were prepared to perform western blotting. (B) Cells were treated with GDC0980: $2.5 \mu \mathrm{M}$ for HPV-positive cell lines (SCC154 and SCC104) and $1 \mu \mathrm{M}$ for HPV-negative cells (SQD9 and SCC61). Control condition cells are treated with equal concentrations of DMSO. GDC0980 was administered $2 \mathrm{~h}$ before radiotherapy ( $6 \mathrm{~Gy})$. After $24 \mathrm{~h}$ of drug treatment, total cell lysates were prepared to perform western blotting. Densitometry quantification was done and relative expression levels were corrected to the loading control and to the non-phosphorylated protein. Uncropped images are provided in the supplementary information. 
A

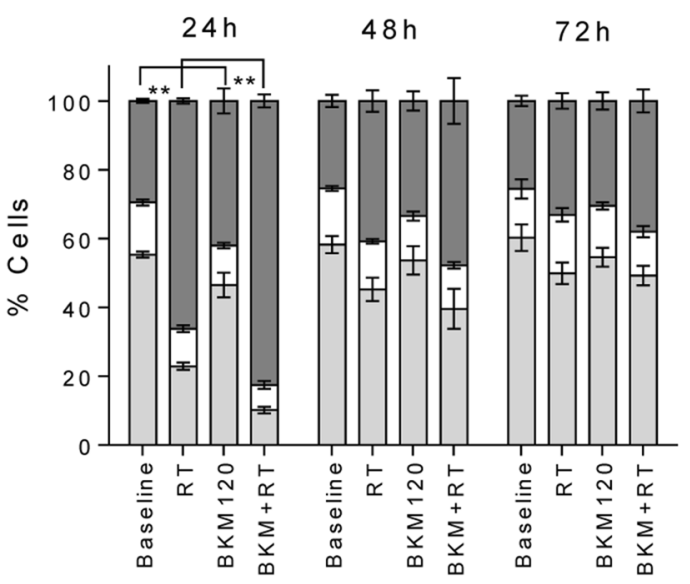

B

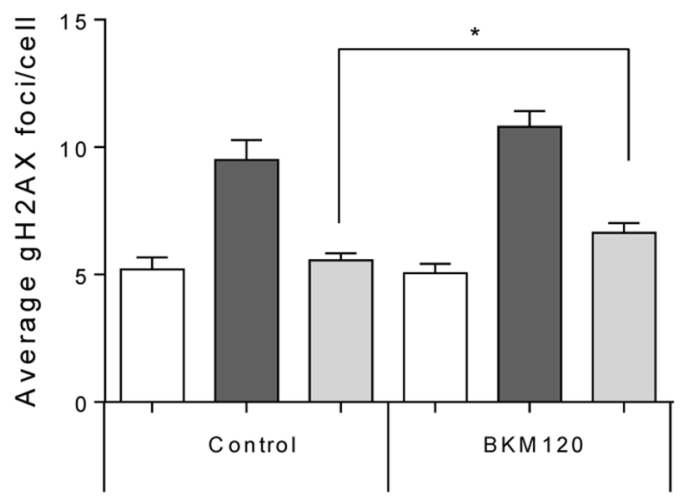

SQD 9

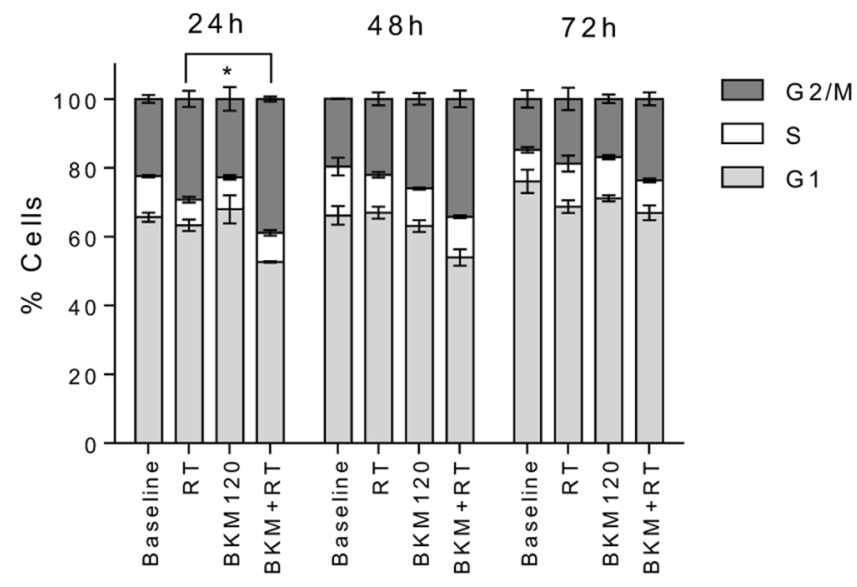

SQD 9

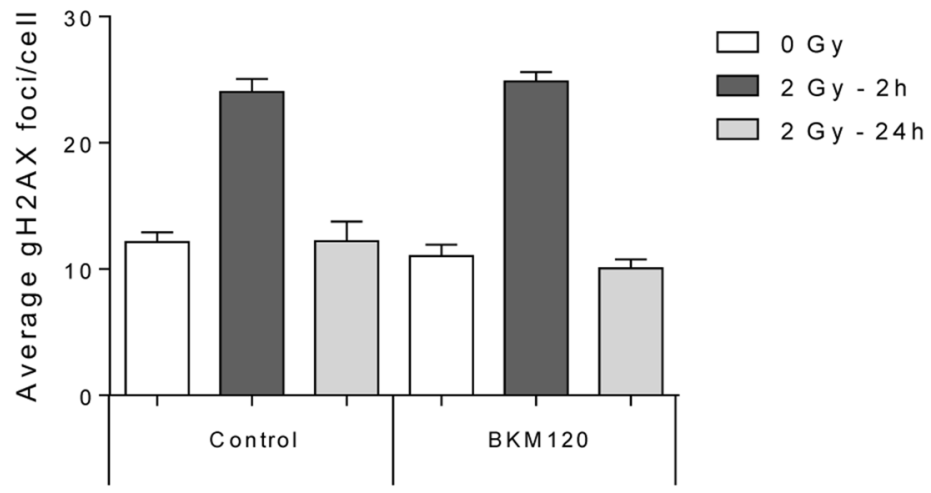

Figure 4. (A) The effect of BKM120 on the cell cycle. The percentage (\%) of SCC104 HPV-positive (left) and SQD9 HPV-negative (right) HNSCC cells distributed in cell cycle phases after treatment for $24 \mathrm{~h}$ with $1 \mu \mathrm{M}$ BKM120 and/or 6 Gy. Data are represented as the mean \pm SEM for three independent performed experiments. One-way Anova plus posthoc t-testing with Bonferroni correction showed the statistical differences. (B) The effect of PI3K inhibition on the DNA damage response. (A) HPV-positive (SCC104) and HPV-negative (SQD9) cells were exposed to $1 \mu \mathrm{M}$ of BKM120 and/or 6 Gy of radiotherapy to assess gH2AX foci. Fixation was done after 2 or $24 \mathrm{~h}$ to represent kinetics of DNA repair. Data are represented as the mean \pm SEM for three independent performed experiments. T-testing showed the significant difference $(\mathrm{p}=0.0454)$.

In line with our gH2AX data, limited but significant G2/M arrest was seen in the combination treatment arm for both HPV-positive and HPV-negative cells. After $72 \mathrm{~h}$, the cells rendered back to baseline conditions in both the RT as well as the combination treatment condition due to further doubling of the cells and DNA repair. Comparing the combination treatment with RT alone, no residual additional differences were seen. G2/M arrest might result from persistent G2/M checkpoint activation caused by unrepaired double strand breaks and can have a protective function providing additional repair time for the cells before entering mitosis ${ }^{56}$. G2/M arrest after RT in HPV-positive cells is extensively documented and has been correlated with increased RT sensitivity of these cells ${ }^{56,59,60}$. PI3K inhibition-related G2/M arrest has been documented in glioblastoma and gastrointestinal carcinoma cells and can be explained by the fact that several important proteins in the regulation of the G2/M cell cycle checkpoint (e.g. Chk1, Wee1) have been identified as targets of PI3K $\mathrm{K}^{19,21,61-63}$. Moreover, in PTEN-loss cells it was shown recently that AKT inhibition restored the relocalization of Chk1 in the nucleus, which rescued G2/M checkpoint activation so that DNA damage can be repaired ${ }^{64}$.

When we have a look at the results of clinical trials in HNSCC, the success of PI3K inhibitors is underwhelming with overall response rates of only $18 \%{ }^{18,65}$. The low clinical durability and resistance to PI3K inhibitors have repeatedly been reported in literature ${ }^{14}$. The activation of rescue pathways can be a part of the answer to this ${ }^{44,45,65,66}$. In addition, the half maximum inhibitory concentration (IC50) of BKM120 to fully block all PI3K forms as monotherapy is high, leading to cellular toxicity via disrupting microtubule dynamics ${ }^{11,21,67,68}$. Moreover, toxicity to normal tissues has been observed with PI3K inhibitors due to broad-spectrum inhibition of multiple signalling pathways ${ }^{7}$. Finally, it is known that PI3K inhibition leads to cytostatic effects and are not cytotoxic which means that tumour regression is not achieved ${ }^{7,32,65}$. However, results of a randomised phase 2 study suggest that BKM120 combined with Paclitaxel could be an effective second-line treatment for patients 
with platinum-pretreated recurrent or metastatic $\mathrm{HNSCC}^{69}$. In addition, it was shown that patients with TP53 alterations, HPV-negative status, low mutational load, or high infiltration of TILs or CD8-positive cells derived survival benefit of the combination of BKM120 and Paclitaxel $^{70}$. Recently, the BURAN phase 3 trial has been initiated to investigate this further. Of four clinical trials that are ongoing with BKM120 in HNSCC, only one (NCT02113878) is assessing the effectiveness in combination with RT of which the results are eagerly awaited.

In conclusion, we showed that the both PI3K inhibitors, BKM120 and GDC0980, induced antiproliferative effects and effectively inhibited the pathway though they did not enhance the RT response in HNSCC cells.

\section{Materials and methods}

Cell culture. The SCC154 cell line was purchased from the German collection of micro-organisms and cell cultures (DSMZ, Germany). Cell lines SCC104 and SCC47 were granted from Dr. Carey Thomas (Michigan University). All three HPV-positive cells lines were cultured and maintained in minimum essential media (MEM, Thermofisher scientific) supplemented with 10\% fetal bovine serum (FBS), $1 \%$ nonessential amino acids and $1 \%$ L-glutamine. The SQD9, SCC61 and CAL27 cell lines were a generous gift of Dr. A. Begg, the Netherlands Cancer Institute (Amsterdam, the Netherlands). These four HPV-negative cell lines were cultured and maintained in Dulbecco's Modified Eagle's Medium (DMEM, Thermofisher scientific) containing 10\% FBS and 1\% sodium pyruvate (Life technologies). Cells were incubated at $37^{\circ} \mathrm{C}$ and passaged via trypsinization. All cell lines were authenticated by ATCC via short-tandem repeat profiling.

All cell lines were wild-type for exon 5, exon 6, exon 10 and exon 21 of the PIK3CA gene, determined by sanger-sequencing. These regions incorporate the hot spot mutations in both the helical domain (E545K) and the kinase domain (H1047R $)^{71,72}$. Three polymorphisms were detected in exon 10: rs 121913273 only in SCC61, and both rs 587777795 and rs 121913274 in cell lines SCC154, SQD9, SCC61 and CAL27.

Chemicals. Buparlisib (NVP-BKM120) and Apitolisib (GDC0980) were bought from Selleck Chemicals (Houston, TX, USA) and a stock solution of $10 \mathrm{mM}$ was made in dimethylsulfoxide (DMSO).

Sulforhodamine B assay. To evaluate the drug's effects on cell survival and proliferation, cells were seeded in 96-well plates. After attachment overnight on $37^{\circ} \mathrm{C}$, cells were treated with drug concentrations of $0 ; 0.5 ; 1$; $2.5 ; 5 \mu \mathrm{M}$ of BKM120 and $0 ; 0.1 ; 0.25 ; 0.5 ; 1 ; 2.5 ; 5 ; 10 \mu \mathrm{M}$ of GDC0980. Two hours after drug administration plates were irradiated with doses of 0, 2, 4 or 6 Gy using a Baltograph (199 kV, 15 mA, Balteau NDT, Belgium). The drug was replaced by fresh full medium after $72 \mathrm{~h}(\mathrm{~h})$ for BKM120 and $24 \mathrm{~h}$ for GDC0980. The time of exposure has been based on pilot studies ${ }^{32,68,73,74}$. One week after seeding, cells were fixed and stained with sulforhodamine B (SRB, Sigma-Aldrich). The incorporated SRB was liberated from cells in a tris-base solution, and optical densities were determined at $570 \mathrm{~nm}$. Cell survival was determined as the relative absorption of SRB in treated wells in comparison to controls.

Colony assay. Cells were seeded in six-well plates and after attachment overnight, cells were treated with 0.5 and $1 \mu \mathrm{M}$ of BKM120 and 1 or $2.5 \mu \mathrm{M}$ of GDC0980. Two hours after drug exposure cells were irradiated with doses of 0, 2, 4 or 6 Gy. After a 14-21 day incubation period, colonies were stained with $0.4 \%$ crystal violet and survival fractions were determined by counting 50 cells or more with a ColCount (Oxford Optronix, Oxford, United Kingdom) as described ${ }^{75}$. Data was normalized to the plating efficiency of unirradiated, non-drug treated controls and curves were fitted using the LQ model as described ${ }^{37}$. Statistical differences in LQ curves were tested with package CFassay in R studio (RStudio: Integrated Development for R. RStudio, Inc., Boston, MA, USA).

Western blotting. For immunoblotting cells were treated with $1 \mu \mathrm{M}$ of BKM120 and for GDC0980, HPVpositive cells were exposed to $2.5 \mu \mathrm{M}$ and HPV-negative cells to $1 \mu \mathrm{M}$. Drug concentrations for western blotting were chosen according the sensitivity of the cell lines to the inhibitors. Control conditions were treated with equal concentrations of DMSO. Two hours after drug exposure cells were irradiated with 6 Gy. Twenty-four hours after drug administration, cells were lysed in $1 \times$ RIPA buffer containing protease and phosphatase inhibitors (Roche). Protein concentrations were determined using Bradford method with Albumin Bovine Serum (BSA, $1 \mu \mathrm{g} / \mu \mathrm{l}$, Sigma-Aldrich) as described ${ }^{76}$. Equal amounts of protein $(15 \mu \mathrm{g})$ were separated on NUPAGE gels (Thermofisher scientific). After electrophoresis, proteins were transferred onto a polyvinylidene fluoride membrane (Biorad) and the membranes were blocked with 5\% non-fatty dry milk. The blots were incubated overnight on $4{ }^{\circ} \mathrm{C}$ with primary antibodies for AKT (\#9272), phospho-AKT Ser 473 (\#9271), mTOR (\#2972), phospho-mTOR ser2448 (\#2971), p70S6 (\#9202), phospho-p70S6 (\#9205), 4EBP1 (\#9452) and phospho-4EBP1 (\#9459) from Cell signalling technology, Vinculin (Sigma-Aldrich) was used as loading control. After incubation with a secondary antibody, visualization was performed with enhanced chemiluminescence reagent (ECL Ultra, Perkin Elmer) using a chemiluminescence imager (c600 Azure Biosystems, CA, USA). Densitometry analysis was performed using ImageJ2 software. To avoid false results, all densitometry values were normalized to the loading control and according total protein. Thereafter, relative values were calculated by dividing each condition by the control condition, meaning no RT and no inhibitor treatment.

Cell cycle analysis. Cells were seeded and after adherence, cells were treated with $1 \mu \mathrm{M}$ of BKM120 and irradiated with $6 \mathrm{~Gy} 2 \mathrm{~h}$ after drug exposure. At the indicated timepoints cells were fixed with $70 \%$ ethanol and stained with $10 \mu \mathrm{g} / \mathrm{ml}$ propidium iodide containing $100 \mu \mathrm{g} / \mathrm{ml}$ RNAse A as described ${ }^{77}$. Cells cycle distribution was assessed by FACSverse (BD Biosciences, CA, USA). 
Immunofluorescence. As described ${ }^{77}$, cells were plated on $\mu$ Clear 96-well plates (Greiner Bio-one) and treated with $1 \mu \mathrm{M}$ of BKM120 and irradiated with $2 \mathrm{~Gy} 2 \mathrm{~h}$ after drug administration. Samples were fixed $4 \mathrm{~h}$ and $24 \mathrm{~h}$ after drug exposure with $4 \%$ paraformaldehyde. After permeabilization with methanol, the cells were stained with primary antibody against gH2AX (ser 139) (clone JBW301 Millipore). This was followed by a staining with secondary antibody conjugated to FITC (Life Technologies). The nuclei were counterstained with DAPI (Sigma Aldrich). Immunofluorescence images were acquired with In Cell Analyzer 2000 (GE Healthcare, IL, USA).

Statistical analysis. All experiments were conducted in triplicate at different time points. Statistical analysis was performed two-sided using GraphPad Prism version 5.00 for Windows (GraphPad Software, La Jolla California, SD, USA). All tests were considered as statistically significant for $\mathrm{p}<0.05\left(^{\star}\right), \mathrm{p}<0.01\left(^{* *}\right)$ and $\mathrm{p}<0.001$ $\left({ }^{* *}\right)$. In the figures, each value is represented as the mean \pm SEM.

Received: 4 May 2020; Accepted: 7 August 2020

Published online: 01 October 2020

\section{References}

1. Bray, F. et al. Global cancer statistics 2018: GLOBOCAN estimates of incidence and mortality worldwide for 36 cancers in 185 countries. CA. Cancer J. Clin. 68, 394-424 (2018).

2. Hashibe, M. et al. Alcohol drinking in never users of tobacco, cigarette smoking in never drinkers, and the risk of head and neck cancer: Pooled analysis in the international head and neck cancer epidemiology consortium. J. Natl. Cancer Inst. 99, 777-789 (2007).

3. Spence, T., Bruce, J., Yip, K. W. \& Liu, F. F. HPV associated head and neck cancer. Cancers (Basel).8, 75 (2016).

4. Lo Nigro, C., Denaro, N., Merlotti, A. \& Merlano, M. Head and neck cancer: Improving outcomes with a multidisciplinary approach. Cancer Manag. Res.9, 363-371 (2017).

5. Forastiere, A., Koch, W., Trotti, A. \& Sidransky, D. Head and neck cancer. N. Engl. J. Med. 345, 1890-1900 (2001).

6. Perri, F. et al. Radioresistance in head and neck squamous cell carcinoma: Biological bases and therapeutic implications. Head Neck 37, 763-770 (2015).

7. Toulany, M. \& Rodemann, H. P. Phosphatidylinositol 3-kinase/Akt signaling as a key mediator of tumor cell responsiveness to radiation. Semin. Cancer Biol. 35, 180-190 (2015).

8. Horn, D., Hess, J., Freier, K., Hoffmann, J. \& Freudlsperger, C. Targeting EGFR-PI3K-AKT-mTOR signaling enhances radiosensitivity in head and neck squamous cell carcinoma. Expert Opin. Ther. Targets 19, 795-805 (2015).

9. Bussink, J., van der Kogel, A. J. \& Kaanders, J. H. Activation of the PI3-K/AKT pathway and implications for radioresistance mechanisms in head and neck cancer. Lancet Oncol. 9, 288-296 (2008).

10. Holmes, D. PI3K pathway inhibitors approach junction. Nat. Rev. Drug Discov. 10, 563-564 (2011).

11. Cai, Y. et al. Dysregulations in the PI3K pathway and targeted therapies for head and neck squamous cell carcinoma. Oncotarget 5, 1-15 (2017)

12. Network, T. C. G. A. Comprehensive genomic characterization of head and neck squamous cell carcinomas. Nature 517, 576-582 (2015).

13. Glorieux, M., Dok, R. \& Nuyts, S. Novel DNA targeted therapies for head and neck cancers : clinical potential and biomarkers. Oncotarget 8, 81662-81678 (2017).

14. Simpson, D. R., Mell, L. K. \& Cohen, E. E. W. Targeting the PI3K/AKT/mTOR pathway in squamous cell carcinoma of the head and neck. Oral Oncol. 51, 291-298 (2015).

15. Lui, V. W. Y. Y. et al. Frequent mutation of the PI3K pathway in head and neck cancer defines predictive biomarkers. Cancer Discov. 3, 761-769 (2013).

16. Pal, I. \& Mandal, M. PI3K and Akt as molecular targets for cancer therapy: current clinical outcomes. Acta Pharmacol. Sin. 33, 1441-1458 (2012).

17. Estévez, L. G., García, E., Hidalgo, M. \& L. G. Estévez; E. Garcia; M. Hidalgo. Inhibiting the PI3K signaling pathway: buparlisib as a new targeted option in breast carcinoma. Clin Transl Oncol18, 541-549 (2016).

18. Maira, S. M. et al. Identification and characterization of NVP-BKM120, an orally available pan-class I PI3-kinase inhibitor. Mol. Cancer Ther. 11, 317-328 (2012).

19. Brachmann, S. M. et al. Characterization of the mechanism of action of the pan class I PI3K inhibitor NVP-BKM120 across a broad range of concentrations. Mol. Cancer Ther. 11, 1747-1757 (2012).

20. Amrein, L., Shawi, M., Grenier, J., Aloyz, R. \& Panasci, L. The phosphatidylinositol-3 kinase i inhibitor BKM120 induces cell death in B-chronic lymphocytic leukemia cells in vitro. Int. J. Cancer 133, 247-252 (2013).

21. Koul, D. et al. Antitumor activity of NVP-BKM120 - A selective pan class I PI3 kinase inhibitor showed differential forms of cell death based on p53 status of glioma cells. Clin. Cancer Res. 18, 184-195 (2012).

22. Bashash, D. et al. Inhibitor of pan class-I PI3K induces differentially apoptotic pathways in acute leukemia cells: Shedding new light on NVP-BKM120 mechanism of action. Int. J. Biochem. Cell Biol. 79, 308-317 (2016).

23. Salphati, L. et al. Preclinical assessment of the absorption and disposition of the phosphatidylinositol 3-kinase/mammalian target of rapamycin inhibitor GDC-0980 and prediction of its pharmacokinetics and efficacy in human. Drug Metab. Dispos. 40, 1785-1796 (2012).

24. Lehmann, B. D. et al. PIK3CA mutations in androgen receptor-positive triple negative breast cancer confer sensitivity to the combination of PI3K and androgen receptor inhibitors. Breast Cancer Res. 16, 406-420 (2014).

25. Wallin, J. J. et al. GDC-0980 is a novel class I PI3K/mTOR kinase inhibitor with robust activity in cancer models driven by the PI3K pathway. Mol. Cancer Ther. 10, 2426-2436 (2011).

26. Morris, Z. S. \& Harari, P. M. Interaction of radiation therapy with molecular targeted agents. J. Clin. Oncol. 32, 2886-2893 (2014).

27. Yard, B. D. et al. A genetic basis for the variation in the vulnerability of cancer to DNA damage. Nat. Commun.7 (2016).

28. Markham, A. Alpelisib: first global approval. Drugs 79, 1249-1253 (2019).

29. Keam, B. et al. In vitro anticancer activity of PI3K alpha selective inhibitor BYL719 in head and neck cancer. Anticancer Res. 35, 175-182 (2015).

30. Kong, D., Yamori, T., Yamazaki, K. \& Dan, S. In vitro multifaceted activities of a specific group of novel phosphatidylinositol 3-kinase inhibitors on hotspot mutant PIK3CA. Invest. New Drugs 32, 1134-1143 (2014). 
31. Mukohara, T. Pi3k mutations in breast cancer: Prognostic and therapeutic implications. Breast Cancer Targets Ther. 7, 111-123 (2015).

32. Anisuzzaman, A. S. et al. In vitro and in vivo synergistic antitumor activity of the combination of BKM120 and erlotinib in head and neck cancer: Mechanism of apoptosis and resistance. Mol. Cancer Ther. 16, 729-738 (2017).

33. Gonzalez-Angulo, A. M. \& Blumenschein, G. R. Defining biomarkers to predict sensitivity to PI3K/Akt/mTOR pathway inhibitors in breast cancer. Cancer Treat. Rev. 39, 313-320 (2013).

34. Wirtz, E. D., Hoshino, D., Maldonado, A. T., Tyson, D. R. \& Weaver, A. M. Response of head and neck squamous cell carcinoma cells carrying PIK3CA mutations to select targeted therapies. JAMA Otolaryngol. Head Neck Surg. 141, 543-549 (2015).

35. Forbes, S. A. et al. COSMIC: Somatic cancer genetics at high-resolution. Nucleic Acids Res. 45, D777-D783 (2017).

36. Martini, M., Ciraolo, E., Gulluni, F. \& Hirsch, E. Targeting PI3K in cancer: Any good news?. Front. Oncol. 3, 108 (2013).

37. Blas, K. et al. Dual blockade of PI3K and MEK in combination with radiation in head and neck cancer. Clin. Transl. Radiat. Oncol. 11, 1-10 (2018).

38. Leiker, A. J. et al. Radiation enhancement of head and neck squamous cell carcinoma by the dual PI3K/mTOR inhibitor PF-05212384. Clin. Cancer Res. 21, 2792-2801 (2015).

39. Herzog, A. et al. PI3K/mTOR inhibitor PF-04691502 antitumor activity is enhanced with induction of wild-type TP53 in human xenograft and murine knockout models of head and neck cancer. Clin. Cancer Res. 19, 3808-3819 (2013).

40. Zumsteg, Z. S. et al. Taselisib (GDC-0032), a potent $\beta$-sparing small molecule inhibitor of PI3K, radiosensitizes head and neck squamous carcinomas containing activating PIK3CA alterations HHS public access. Clin Cancer Res. Clin Cancer Res. 15, 2009-2019 (2016).

41. Hayman, T. J. et al. The ATP-competitive mTOR inhibitor INK128 enhances in vitro and in vivo radiosensitivity of pancreatic carcinoma cells. Clin. Cancer Res. 20, 110-119 (2014).

42. Liu, W.-L. et al. Targeting phosphatidylinositide3-kinase/Akt pathway by BKM120 for radiosensitization in hepatocellular carcinoma. Oncotarget 5, 3662-3672 (2014).

43. Abazeed, M. E. et al. Integrative radiogenomic profiling of squamous cell lung cancer. Cancer Res. 73, 6289-6298 (2013).

44. Lattanzio, L. et al. Treatment effect of buparlisib, cetuximab and irradiation in wild-type or PI3KCA-mutated head and neck cancer cell lines. Invest. New Drugs 33, 310-320 (2015).

45. Bozec, A. et al. Combination of phosphotidylinositol-3-kinase targeting with cetuximab and irradiation: A preclinical study on an orthotopic xenograft model of head and neck cancer. Head Neck 36, 1391 (2016).

46. Kelly, C. J. et al. Regulation of $\mathrm{O} 2$ consumption by the PI3K and mTOR pathways contributes to tumor hypoxia. Radiother. Oncol. 111, 72-80 (2014).

47. Burrows, N. et al. Phosphatidylinositide 3-kinase (PI3K) and PI3K-related kinase (PIKK) activity contributes to radioresistance in thyroid carcinomas. Oncotarget 7, 63106-63123 (2016).

48. Blas, K. G. et al. Targeted inhibition of PI3K and MEK in combination with radiation therapy in UT15: A murine model of head and neck squamous cell carcinoma. Int. J. Radiat. Oncol. 99, E578-E579 (2017).

49. Fokas, E. et al. Dual inhibition of the PI3K/mTOR pathway increases tumor radiosensitivity by normalizing tumor vasculature. Cancer Res. 72, 239-248 (2012).

50. McGowan, D. R. et al. Buparlisib with thoracic radiotherapy and its effect on tumour hypoxia: A phase I study in patients with advanced non-small cell lung carcinoma. Eur. J. Cancer 113, 87-95 (2019).

51. Sun, Y. et al. In vitro potency of dual PI3K/mTORC1/C2 inhibitor, GDC-0980 in ER+ and HER2 overexpressing breast cancer cells. [abstract]. in Proceedings of the 106th Annual Meeting of the American Association for Cancer Research 2015. Cancer Res.75, 4717 (2015).

52. De, P. et al. Doubling down on the PI3K-AKT-mTOR pathway enhances the antitumor efficacy of PARP inhibitor in triple negative breast cancer model beyond BRCA-ness. Neoplasia 16, 43-72 (2014).

53. Toulany, M. \& Rodemann, H. P. Potential of Akt mediated DNA repair in radioresistance of solid tumors overexpressing erbBPI3K-Akt pathway. Transl. Cancer Res. 2, 190-202 (2013).

54. Holler, M. et al. Dual targeting of Akt and mTORC1 impairs repair of DNA double-strand breaks and increases radiation sensitivity of human tumor cells. PLoS ONE 11, e0154745 (2016).

55. Mueck, K., Rebholz, S., Harati, M. D., Rodemann, H. P. \& Toulany, M. Akt1 stimulates homologous recombination repair of DNA double-strand breaks in a Rad51-dependent manner. Int. J. Mol. Sci. 18, 1-17 (2017).

56. Rieckmann, T. et al. HNSCC cell lines positive for HPV and p16 possess higher cellular radiosensitivity due to an impaired DSB repair capacity. Radiother. Oncol. 107, 242-246 (2013).

57. Weaver, A. N. et al. DNA double strand break repair defect and sensitivity to poly ADP- ribose polymerase (PARP) inhibition in human papillomavirus 16-positive head and neck squamous cell carcinoma. Oncotarget 6, 26995-27007 (2015).

58. Dok, R. et al. P16INK4a impairs homologous recombination-mediated DNA repair in human papillomavirus-positive head and neck tumors. Cancer Res. 74, 1739-1751 (2014).

59. Arenz, A. et al. Increased radiosensitivity of HPV-positive head and neck cancer cell lines due to cell cycle dysregulation and induction of apoptosis. Strahlenther. Onkol. 190, 839-846 (2014).

60. Nickson, C. M., Moori, P., Carter, R. J., Rubbi, C. P. \& Parsons, J. L. Misregulation of DNA damage repair pathways in HPV-positive head and neck squamous cell carcinoma contributes to cellular radiosensitivity. Oncotarget 8, 29963-29975 (2017).

61. Wachsberger, P. R. et al. Hsp90 inhibition enhances PI-3 kinase inhibition and radiosensitivity in glioblastoma. J. Cancer Res. Clin. Oncol. 140, 573-582 (2014).

62. Jane, E. P., Premkumar, D. R., Morales, A., Foster, K. A. \& Pollack, I. F. Inhibition of phosphatidylinositol 3-kinase/AKT signaling by NVP-BKM120 promotes ABT-737-induced toxicity in a caspase-dependent manner through mitochondrial dysfunction and DNA damage response in established and primary cultured glioblastoma cells. J. Pharmacol. Exp. Ther. 350, 22-35 (2014).

63. Mueller, A. et al. Selective PI3K inhibition by BKM120 and BEZ235 alone or in combination with chemotherapy in wild-type and mutated human gastrointestinal cancer cell lines. Cancer Chemother. Pharmacol. 69, 1601-1615 (2012).

64. Mansour, W. Y. et al. Loss of PTEN-assisted G2/M checkpoint impedes homologous recombination repair and enhances radiocurability and PARP inhibitor treatment response in prostate cancer. Sci. Rep. 8, 3947 (2018).

65. Michmerhuizen, N. L., Leonard, E., Kulkarni, A. \& Brenner, J. C. Differential compensation mechanisms define resistance to PI3K inhibitors in PIK3CA amplified HNSCC. Otorhinolaryngol. Head Neck Surg. 1, 44-50 (2016).

66. Ren, H. et al. Blockade efficacy of MEK/ERK-dependent autophagy enhances PI3K/Akt inhibitor NVP-BKM120's therapeutic effectiveness in lung cancer cells. Oncotarget 7, 67277-67287 (2014).

67. Fruman, D. A. \& Rommel, C. PI3K and cancer: Lessons, challenges and opportunities. Nat. Rev. Drug Discov. 13, 140-156 (2014).

68. Kanteti, R. et al. PI3 kinase pathway and MET inhibition is efficacious in malignant pleural mesothelioma. Sci. Rep. 6, 32992 (2016).

69. Soulières, D. et al. Buparlisib and paclitaxel in patients with platinum-pretreated recurrent or metastatic squamous cell carcinoma of the head and neck (BERIL-1): A randomised, double-blind, placebo-controlled phase 2 trial. Lancet Oncol. 18, 323-335 (2017).

70. Soulieres, D. et al. Molecular alterations and buparlisib efficacy in patients with squamous cell carcinoma of the head and neck: Biomarker analysis from BERIL-1. Clin. Cancer Res. 24, 2505-2516 (2018).

71. Du, L., Shen, J., Weems, A. \& Lu, S. L. Role of phosphatidylinositol-3-kinase pathway in head and neck squamous cell carcinoma. J. Oncol. 2012, 450179 (2012). 
72. Ang, D. et al. Novel method for PIK3CA mutation analysis: Locked nucleic acid-PCR sequencing. J. Mol. Diagn. 15, 312-318 (2013).

73. Bohnacker, T. et al. Deconvolution of buparlisib's mechanism of action defines specific PI3K and tubulin inhibitors for therapeutic intervention. Nat. Commun. 8, 1-13 (2017).

74. Rahmani, M. et al. Cotargeting BCL-2 and PI3K induces BAX-dependent mitochondrial apoptosis in AML cells. Cancer Res. 78, 3075-3086 (2018).

75. Gonnissen, A. et al. The hedgehog inhibitor GANT61 sensitizes prostate cancer cells to ionizing radiation both in vitro and in vivo. Oncotarget 7, 84286-84298 (2016).

76. Dok, R. et al. Nuclear p16INK4a expression predicts enhanced radiation response in head and neck cancers. Oncotarget 7, 3878538795 (2016).

77. Dok, R. et al. Radiosensitization approaches for HPV-positive and HPV-negative head and neck squamous carcinomas. Int. J. Cancer 146, 1075-1085 (2020).

\section{Acknowledgements}

We thank the support of Dr. Agnieszka Wozniak for the analysis of the Sanger sequencing data. We also appreciate the help of Thorvald Dox and Margaux Faes with the statistical analysis in R of the colony assays.

\section{Author contributions}

M.G., R.D. and S.N. contributed to the conception and design of the article. M.G. and R.D. contributed to the acquisition and interpretation of data. M.G. wrote the paper. All authors contributed critical revision and final approval of the article. All authors are accountable for the content of this manuscript.

\section{Funding}

This research was supported by the Fonds Wetenschappelijk Onderzoek, Kom Op Tegen Kanker and RD is supported by Emmanuel van der Schueren fellowship for postdoctoral researchers from Kom op Tegen Kanker.

\section{Competing interests}

The authors declare no competing interests.

\section{Additional information}

Supplementary information is available for this paper at https://doi.org/10.1038/s41598-020-73249-z.

Correspondence and requests for materials should be addressed to S.N.

Reprints and permissions information is available at www.nature.com/reprints.

Publisher's note Springer Nature remains neutral with regard to jurisdictional claims in published maps and institutional affiliations.

(c) (i) Open Access This article is licensed under a Creative Commons Attribution 4.0 International License, which permits use, sharing, adaptation, distribution and reproduction in any medium or format, as long as you give appropriate credit to the original author(s) and the source, provide a link to the Creative Commons licence, and indicate if changes were made. The images or other third party material in this article are included in the article's Creative Commons licence, unless indicated otherwise in a credit line to the material. If material is not included in the article's Creative Commons licence and your intended use is not permitted by statutory regulation or exceeds the permitted use, you will need to obtain permission directly from the copyright holder. To view a copy of this licence, visit http://creativecommons.org/licenses/by/4.0/.

(C) The Author(s) 2020 